\title{
Metric structures on fibered manifolds through partitions of unity
}

\author{
Hulya Kadioglu ${ }^{1}$ and Robert Fisher $\mathrm{Jr}^{2}$ \\ ${ }^{1}$ Yildiz Technical University, Esenler, Istanbul, Turkey \\ ${ }^{1,2}$ Department of Mathematics, Idaho State University, Pocatello, USA
}

Received: 29 March 2016, Revised: 2 April 2016, Accepted: 10 April 2016

Published online: 10 May 2016.

\begin{abstract}
The notion of partitions of unity is extremely useful as it allows one to extend local constructions on Euclidean patches to global ones. It is widely used in many fields in mathematics. Therefore, prolongation of this useful tool to another manifold may help constructing many geometric structures. In this paper, we construct a partition of unity on a fiber bundle by using a given partition of unity on the base manifold. On the other hand we show that the converse is also possible if it is a vector bundle. As an application, we define a Riemannian metric on the fiber bundle by using induced partition of unity on the fiber bundle.
\end{abstract}

Keywords: Partitions of Unity, fiber bundles, Riemannian metric.

\section{Introduction}

A partition of unity is a useful tool that helps us work in local coordinates. This can be a tricky matter when we are doing things all over our manifold, since it is almost never the case that the entire manifold fits into a single coordinate patch. A (smooth) partition of unity is a way of breaking the function with the constant value 1 up into a bunch of (smooth) pieces that will be easier to work with.

Partitions of unity is widely used in many fields of mathematics, most notably in many branches of Geometry and Analysis, such as defining integration on manifolds and Riemannian metric on an arbitrary manifold (see [2], Prop. 5.1.8). Other geometric applications include section extension property (SEP) on topological bundles, and fiber homotopy equivalence for fibrations [1]. (See [3], [6],[7] for further applications.)

More specifically, a partition of unity subordinate to an open cover $\left\{U_{\alpha}\right\}$ is a collection of non-negative smooth functions $\psi_{\alpha}: M \rightarrow \mathbb{R}$ such that

1. For all $\alpha, \operatorname{Supp}\left\{\psi_{\alpha}\right\} \subset U_{\alpha}$ for some $\alpha$ (here $\operatorname{Supp}\left\{\psi_{\alpha}\right\}$ is the closure of the subset where $\psi_{\alpha}(x) \neq 0$ ).

2. $0 \leq \psi_{\alpha}(x) \leq 1$ on $M$.

3. Set of supports $\left\{\operatorname{Supp}\left\{\psi_{\alpha}\right\}\right\}_{\alpha \in I}$ is a locally finite set. That is $\forall x \in M$, there is an open neighborhood $V_{x}$ of $x$ such that there exist only finitely many $\psi_{\alpha}$ with $\operatorname{Supp}\left\{\psi_{\alpha}\right\} \cap V_{x} \neq \emptyset$.

4. $\sum_{\alpha} \psi_{\alpha}(x)=1$ (this sum is finite because of 3 ).

For any manifold $M$ with atlas $\Omega=\left\{\left(U_{\alpha}, \phi_{\alpha}\right)\right\}$, there exists a Riemannian metric $g_{p}$ on $T_{p} M$ such that

$$
g_{p}\left(X_{p}, Y_{p}\right)=\sum_{\alpha \in I} \psi_{\alpha}(p)<X_{p}, Y_{p}>^{\alpha}
$$


where $p \in M$, and $\left\{\psi_{\alpha}\right\}$ is a partition of unity subordinate to $\Omega$. Here for each $\alpha$, we label $<,>^{\alpha}$ as the usual dot product with respect to the coordinate basis over $U_{\alpha}$.

In this paper, we suppose that all manifolds are real, and that manifolds are smooth and finite-dimensional. We shall require the topology on each manifold to be Hausdorff, second-countable, and connected. It follows from these assumptions that our manifolds admit partitions of unity.

Organization: In section 2, we define a partition of unity on $\mathbb{E}$ by using an arbitrary partition of unity on $M$, which we call induced partition of unity. We also show that one can construct a partition of unity on $M$ by using a partition of unity on a vector bundle $\mathbb{E}$. In section 3, we use the induced partition of unity to define a Riemannian metric on $\mathbb{E}$. We call this metric as the induced metric on $\mathbb{E}$.

\section{Induced partition of unity}

In this section, we give some theorems/definitions to define an induced partition of unity on a fiber bundle $(\mathbb{E}, \pi, M, F)$.

Lemma 1. Suppose that $f: M \rightarrow \mathbb{R}$ be a function, $\pi: \mathbb{E} \rightarrow M$ be a surjective map, and $\Delta=\{x \in M: f(x) \neq 0\}$, and $\Delta^{\prime}=\{h \in \mathbb{E}:(f \circ \pi)(h) \neq 0\}$. Then $\pi\left(\Delta^{\prime}\right)=\Delta$.

Proof. Assume that $x \in \pi\left(\Delta^{\prime}\right)$, then

$$
x=\pi(h), \quad(f \circ \pi)(h) \neq 0,
$$

which implies that

$$
x \in M, \quad f(x) \neq 0 .
$$

Then $x \in \Delta$, which shows that $\pi\left(\Delta^{\prime}\right) \subset \Delta$.

Assume that $x \in \Delta$. Then we have

$$
x \in M, \quad f(x) \neq 0 .
$$

Since $\pi$ is a surjective map, then there exists $h \in \mathbb{E}$ such that $x=\pi(h)$. Therefore $h \in \pi^{-1}(x)$ and $f(x) \neq 0$.

$$
\begin{aligned}
& h \in \pi^{-1}(x), \quad f(\pi(h)) \neq 0 \\
& h \in \mathbb{E}, \quad(f \circ \pi)(h) \neq 0 \\
& h \in \Delta^{\prime}, \quad \pi(h)=x \\
& x \in \pi\left(\Delta^{\prime}\right),
\end{aligned}
$$

which shows that $\Delta \subset \pi\left(\Delta^{\prime}\right)$.

Remark 1. If additionally, the function $\pi$ in Lemma 1 is continuous, then there exists a following relation between supports of functions $f$ and $f \circ \pi$

$$
\operatorname{supp}\{f\}=\pi(\operatorname{supp}\{f \circ \pi\}) .
$$

Theorem 1. Let $\mathbb{E}$ be a fibered manifold with base space $M$. If $\left\{\psi_{\alpha}\right\}$ is a smooth partition of unity of $M$ subordinate to its atlas $\left\{\left(U_{\alpha}, \phi_{\alpha}\right)\right\}$, then $\left\{\psi_{\alpha} \circ \pi\right\}$ is a smooth partition of unity of $\mathbb{E}$ subordinate to the open cover $\left\{\left(\pi^{-1}\left(U_{\alpha}\right)\right\}\right.$. Moreover, if $\mathbb{E}$ is a fiber bundle, then $\left\{\psi_{\alpha} \circ \pi\right\}$ is a smooth partition of unity subordinate to the atlas $\left\{\left(\pi^{-1}\left(U_{\alpha}\right),\left(\phi_{\alpha} \times \eta\right) \circ \Phi_{\mathbb{E}}\right)\right\}$ of $\mathbb{E}$. 
Proof. We begin our proof by showing that each support is a subset of $\pi^{-1}\left(U_{\alpha}\right)$. Since $\left\{\psi_{\alpha}\right\}$ is a partition of unity subordinate to $\left\{U_{\alpha}\right\}$, then for each $\alpha, \operatorname{supp}\left\{\psi_{\alpha}\right\} \subset U_{\alpha}$. Moreover, since $\pi$ is a surjective continuous function, then from Remark 1, we have

$$
\operatorname{supp}\left\{\psi_{\alpha}\right\}=\pi\left(\operatorname{supp}\left\{\psi_{\alpha} \circ \pi\right\}\right),
$$

which is a subset of $U_{\alpha}$, that is $\pi(\operatorname{supp}\{\psi \circ \pi\}) \subset U_{\alpha}$. This implies $\operatorname{supp}\left\{\psi_{\alpha} \circ \pi\right\} \subset \pi^{-1}\left(U_{\alpha}\right)$.

The second condition is obvious, since $\operatorname{Im}\left(\psi_{\alpha} \circ \pi\right) \subset \operatorname{Im}\left(\psi_{\alpha}\right)$, then we have

$$
0 \leq\left(\psi_{\alpha} \circ \pi\right)(h) \leq 1 .
$$

The equations comes from the surjectiveness of the function $\pi$.

To show the third condition to hold, we show that there exists finite number of index $\alpha$ such that $\forall h \in \mathbb{E}$, $\left(\psi_{\alpha} \circ \pi\right)(h) \neq 0$.

Let

$$
I_{x}^{\prime}=\left\{\alpha \in I: \operatorname{Supp}\left\{\psi_{\alpha}\right\} \cap V_{x} \neq \emptyset\right\}
$$

and

$$
I_{h}^{\prime \prime}=\left\{\alpha \in I: \operatorname{Supp}\left\{\psi_{\alpha} \circ \pi\right\} \cap W_{h} \neq \emptyset\right\}
$$

where for all $x \in M, V_{x}$ represents an open neigbourhood of $x$, and $W_{h}=\pi^{-1}\left(V_{x}\right)$ with $\pi(h)=x$. Since $\pi$ is a continuous function, then $W_{h}$ is an open neighborhood of $h$. Since $\left\{\psi_{\alpha}\right\}$ is a partition of unity, then $I_{x}^{\prime}$ is a finite set.

Suppose that $\alpha \in I_{h}^{\prime \prime}$, then

$$
\begin{gathered}
\exists \tilde{h} \in \operatorname{Supp}\left\{\psi_{\alpha} \circ \pi\right\} \cap W_{h} \\
\pi(\tilde{h}) \in \pi\left(\operatorname{Supp}\left\{\psi_{\alpha} \circ \pi\right\}\right) \text { and } \pi(\tilde{h}) \in \pi\left(W_{h}\right) \subset V_{x} .
\end{gathered}
$$

By Remark 1, we have

$$
\begin{gathered}
\pi(\tilde{h}) \in \operatorname{Supp}\left\{\psi_{\alpha}\right\} \text { and } \pi(\tilde{h}) \in V_{x} \\
\operatorname{Supp}\left\{\psi_{\alpha}\right\} \cap V_{x} \neq \emptyset \\
\alpha \in I_{x}^{\prime},
\end{gathered}
$$

which implies that $I_{h}^{\prime \prime} \subset I_{x}^{\prime}$ for all $h \in \mathbb{E}$, and $\pi(h)=x \in M$. Since $I_{x}^{\prime}$ is a finite, then $I_{h}^{\prime \prime}$ is also finite. Thus set of supports of $\left\{\psi_{\alpha} \circ \pi\right\}$ is locally finite set.

To show the last condition, we should show that the sum of all elements in the image of $\psi_{\alpha} \circ \pi$ is 1 . Since $\pi$ is a surjective map, there exists $x \in M$ such that $\pi(h)=x$ for all $h \in \mathbb{E}$. Then $\forall h \in \mathbb{E}$

$$
\sum_{\alpha \in I}\left(\psi_{\alpha} \circ \pi\right)(h)=\sum_{\alpha \in I} \psi_{\alpha}(x)=1
$$

which implies that $\left\{\psi_{\alpha} \circ \pi\right\}$ is a partition of unity subordinate to the open cover $\pi^{-1}\left(U_{\alpha}\right)$. Since $\left\{\psi_{\alpha}\right\}$ is smooth, then $\left\{\psi_{\alpha} \circ \pi\right\}$ is also smooth. If $\mathbb{E}$ is a fiber bundle, then for every atlas $\left\{\left(U_{\alpha}, \phi_{\alpha}\right)\right\}$, there exists an adapted coordinate system 
([8], Prop 1.1.14)

$$
y_{\alpha}: \pi^{-1}\left(U_{\alpha}\right) \rightarrow U_{\alpha} \times V_{\alpha}
$$

defined by

$$
y_{\alpha}=\left(\phi_{\alpha} \times \eta_{\alpha}\right) \circ \Phi_{\mathbb{E}},
$$

where $V_{\alpha}=\left(p r_{2} \circ \Phi_{\mathbb{E}}\right)\left(\pi^{-1}\left(U_{\alpha}\right)\right)$. Therefore $\left\{\psi_{\alpha} \circ \pi\right\}$ is a partition of unity subordinate to adapted atlas $\left\{\left(\pi^{-1}\left(U_{\alpha}\right), y_{\alpha}\right)\right\}$. By above theorem define a partition of unity on $\mathbb{E}$ which is induced by any partition of unity subordinate to $\Omega$. We recall that the induced partition of unity is the set of pull-back functions $\pi^{*} \psi_{\alpha}$. The natural question is in which conditions the converse statement holds. Next theorem takes care of this issue.

Theorem 2. If $(\mathbb{E}, \pi, M, F)$ is a vector bundle, then every partition of unity $\left\{\widetilde{\psi}_{\alpha}\right\}$ on $\mathbb{E}$ induce a pull-back partition of unity $s^{*} \widetilde{\psi}_{\alpha}$ on $M$, where $s \in \Gamma(\pi)$.

Proof. Suppose that $\left\{\widetilde{\psi}_{\alpha}\right\}$ be a partition of unity subordinate to $\pi^{-1}\left(U_{\alpha}\right)$. Since $\mathbb{E}$ is a vector bundle, there exists a (global) section $s \in \Gamma(\pi)$. To show the condition 1, suppose that $I$ be an index set, and let $A=\left\{h \in \mathbb{E}: \widetilde{\psi}_{\alpha}(h) \neq 0\right\}$ (therefore $\left.\bar{A}=\operatorname{Supp}\left\{\widetilde{\psi}_{\alpha}\right\}\right)$, and $A^{\prime}=\left\{x \in M:\left(\widetilde{\psi}_{\alpha} \circ s\right)(x) \neq 0\right\}$. Then

$$
\pi(A)=\left\{\pi(h): \widetilde{\psi}_{\alpha}(h) \neq 0\right\}
$$

If $x \in A^{\prime}$ then

$$
\begin{gathered}
\left(\widetilde{\psi}_{\alpha} \circ s\right)(x) \neq 0 \\
\widetilde{\psi}_{\alpha}(h) \neq 0, \quad s(x)=h \\
\widetilde{\psi}_{\alpha}(h) \neq 0, \quad x=\pi(h) \\
x \in \pi(A)
\end{gathered}
$$

which leads to $A^{\prime} \subset \pi(A)$. On the other hand, since $\pi$ is a continuous function, then $\bar{A}^{\prime} \subset \pi(\bar{A})$. Since $\left\{\widetilde{\psi}_{\alpha}\right\}$ is a partition of unity subordinate to $\pi^{-1}\left(U_{\alpha}\right)$, then $\bar{A}=\operatorname{Supp}\left\{\widetilde{\psi}_{\alpha}\right\} \subset \pi^{-1}\left(U_{\alpha}\right)$. Therefore

$$
\operatorname{Supp}\left\{s^{*}\left(\widetilde{\psi}_{\alpha}\right)\right\}=\bar{A}^{\prime} \subset \pi(\bar{A}) \subset U_{\alpha} .
$$

For the third condition, suppose that

$$
I_{x}=\left\{\alpha \in I: \exists V_{x} \in N(x), \operatorname{Supp}\left\{s^{*} \widetilde{\psi}_{\alpha}\right\} \cap V_{x} \neq \emptyset\right\},
$$

here, $N(x)$ represents the neighborhoods of $x$. To show $\left\{s^{*} \widetilde{\psi}_{\alpha}\right\}$ is locally finite, we need to show that $I_{x}$ is finite for all $x \in M$.

Suppose that $A_{\alpha}^{\prime}=\left\{y \in M: s^{*}\left(\widetilde{\psi}_{\alpha}\right)(y) \neq 0\right\}$, then $\operatorname{Supp}\left\{s^{*} \widetilde{\psi}_{\alpha}\right\}=\overline{A_{\alpha}^{\prime}}$. Therefore, $\forall \alpha \in I_{x}$, there exists an open neighborhood $V_{x}$ of $x$ such that

$$
\begin{gathered}
\exists z \in V_{x} \cap \overline{A_{\alpha}^{\prime}} \\
s(z) \in s\left(V_{x}\right), \text { and } s(z) \in \overline{s\left(A_{\alpha}^{\prime}\right)} \\
s(z) \in \pi^{-1}\left(V_{x}\right), \quad s(z) \in \overline{s\left(A_{\alpha}^{\prime}\right)} \\
\pi^{-1}\left(V_{x}\right) \cap \overline{s\left(A_{\alpha}^{\prime}\right)} \neq \emptyset .
\end{gathered}
$$


Since $\pi$ is a continuous function, and $V_{x}$ is open, then $\pi^{-1}\left(V_{x}\right)$ is an open. On the other hand since $s(x) \in s\left(V_{x}\right) \subset \pi^{-1}\left(V_{x}\right)$, then $\pi^{-1}\left(V_{x}\right)$ is an open neighborhood of $s(x)$.

So far, we have shown that there exists an open neighborhood $\pi^{-1}\left(V_{x}\right)$ of $s(x)$ such that

$$
\pi^{-1}\left(V_{x}\right) \cap \overline{s\left(A_{\alpha}^{\prime}\right)} \neq \emptyset
$$

On the other hand, suppose that $z^{\prime} \in s\left(A_{\alpha}^{\prime}\right)$. Then there exists a $z \in A_{\alpha}^{\prime}$ such that $z^{\prime}=s(z)$. Then $\psi_{\alpha}\left(z^{\prime}\right)=\left(\psi_{\alpha} \circ s\right)(z) \neq 0$, which implies that $z^{\prime} \in A_{\alpha}$. Since $s$ is a continuous function, then $\overline{s\left(A_{\alpha}^{\prime}\right)} \subset \overline{A_{\alpha}}$. From Equation 3, we have

$$
\pi^{-1}\left(V_{x}\right) \cap \overline{A_{\alpha}} \neq \emptyset
$$

which shows that $\alpha \in \widetilde{I}_{s(x)}$. Therefore $I_{x} \subset \widetilde{I}_{s(x)}$. Since $\widetilde{\psi}_{\alpha}$ is a partition of unity, then for all $h \in \mathbb{E}, \widetilde{I}_{h}$ is finite. Since $s(x) \in \mathbb{E}$, then $\widetilde{I}_{s(x)}$ is finite, which implies that $I_{x}$ is finite. So, set of supports $\operatorname{Supp}\left\{\widetilde{\psi}_{\alpha} \circ s\right\}$ is locally finite. Thus, condition 3 holds. Conditions 2 and 4 can be shown easily. Then, $\left\{\widetilde{\psi}_{\alpha} \circ s\right\}$ is a partition of unity subordinate to $\left\{U_{\alpha}\right\}$.

Corollary 1. If $(\mathbb{E}, \pi, M, F)$ is a fiber bundle, and if $\left\{\psi_{\alpha}\right\}$ is a partition of unity subordinate to an atlas on $M$, then for all $x \in M$, there exists a partition of unity $\left\{\bar{\psi}_{\alpha}\right\}$ subordinate to an atlas $\Theta=\left\{\left(V_{\alpha}, \eta_{\alpha}\right)\right\}$ on $F$ with the property that $\bar{\psi}_{\alpha}(v)=\psi_{\alpha}(x)$, where $v \in \gamma_{x}\left(\pi^{-1}\{x\}\right)$ and $V_{\alpha}=\left(p r_{2} \circ \Phi_{\mathbb{E}}\right)\left(\pi^{-1}\left(U_{\alpha}\right)\right)$.

Proof. If $(\mathbb{E}, \pi, M, F)$ is a fiber bundle, then for all $x \in M$, there exists a diffeomorphism $\gamma_{x}: \pi^{-1}\{x\} \rightarrow F$ (which leads $\left(\gamma_{x}\right)^{-1}$ being a diffeomorphism) and if $\left\{\psi_{\alpha}\right\}$ is a partition of unity subordinate to an atlas $\left\{\left(U_{\alpha}, \phi_{\alpha}\right)\right\}$, then by Theorem $1,\left\{\psi_{\alpha} \circ \pi\right\}$ is a partition of unity subordinate to an adapted atlas $\left.\left\{\pi^{-1}\left(U_{\alpha}\right), y_{\alpha}\right)\right\}$, where $y_{\alpha}$ is defined by Equation 2 . Suppose that $\bar{\psi}_{\alpha}=\psi_{\alpha} \circ \pi \circ \gamma_{x}^{-1}$ for all $x \in M$. Since $\left(\gamma_{x}\right)^{-1}$ is a diffeomorphism (therefore it is surjective and smooth), then $\bar{\psi}_{\alpha}$ is also a partition of unity subordinate to the atlas $\left\{\left(V_{\alpha}, \eta_{\alpha}\right)\right\}$. Also, for all $v \in F$,

$$
\begin{aligned}
\bar{\psi}_{\alpha}(v) & =\left(\psi_{\alpha} \circ \pi \circ \gamma_{x}^{-1}\right)(v) \\
& =\left(\psi_{\alpha} \circ \pi\right)\left(\Phi_{\mathbb{E}}^{-1}(x, v)\right) \\
& =\left(\psi_{\alpha} \circ p r_{1}\right)(x, v) \\
& =\psi_{\alpha}(x),
\end{aligned}
$$

where $v \in \gamma_{x}\left(\pi^{-1}\{x\}\right)$, which finishes the proof.

Definition 1. We call $\left\{\psi_{\alpha} \circ \pi\right\}$ and $\left\{\bar{\psi}_{\alpha}\right\}$ as induced partitions of unity on $\mathbb{E}$ and $F$ respectively.

\section{Riemannian Metrics}

We now define the induced Riemannian metrics on $\mathbb{E}$ and $F$, by the above partition of unities on $\mathbb{E}$ and $F$ respectively, which are defined in Section 2.

Theorem 3. Suppose that $(\mathbb{E}, \pi, M, F)$ be a fiber bundle. By Equation 1, the induced Riemannian metric $g_{\mathbb{E}}$ on $\mathbb{E}$ (by partition of unity subordinate to adapted atlas) is defined as follows:

$$
g_{\mathbb{E}}(\bar{V}, \bar{W})=g_{M}\left(\pi_{*}(\bar{V}), \pi_{*}(\bar{W})+g_{F}\left(\gamma_{*}(\bar{V}), \gamma_{*}(\bar{W})\right)\right.
$$

Here, $g_{F}$ is the metric, constructed by the induced partition unity $\left\{\bar{\psi}_{\alpha}\right\}$. 
Proof.

$$
y_{\alpha}=\left(\phi_{\alpha} \times \eta_{\alpha}\right) \circ \Phi_{\mathbb{E}}=\left(\phi_{\alpha} \circ \pi, \eta_{\alpha} \circ p r_{2} \circ \Phi_{\mathbb{E}}\right) .
$$

To define the induced metric $g_{\mathbb{E}}$ on $\mathbb{E}$, we first compute $<,>_{h}^{\alpha}$ for all $h \in \mathbb{E}$. For all $\bar{V}_{h}, \bar{W}_{h} \in T_{h} \mathbb{E}$,

$$
<\bar{V}_{h}, \bar{W}_{h}>_{h}^{\alpha}=<\left(y_{\alpha}\right)_{*}(\bar{V}),\left(y_{\alpha}\right)_{*}(\bar{W})>
$$

By using Equation 5 we have

$$
\begin{aligned}
& <\bar{V}_{h}, \bar{W}_{h}>_{h}^{\alpha} \\
& =<\left(\left(\phi_{\alpha} \circ \pi\right)_{*}\left(\bar{V}_{h}\right),\left(\eta_{\alpha} \circ p r_{2} \circ \Phi_{\mathbb{E}}\right)_{*}\left(\bar{V}_{h}\right)\right),\left(\left(\phi_{\alpha} \circ \pi\right)_{*}\left(\bar{W}_{h}\right),\left(\eta_{\alpha} \circ p r_{2} \circ \Phi_{\mathbb{E}}\right)_{*}\left(\bar{W}_{h}\right)\right)> \\
& =<\left(\phi_{\alpha}\right)_{*}\left(\pi_{*}\left(\bar{V}_{h}\right)\right),\left(\phi_{\alpha}\right)_{*}\left(\pi_{*}\left(\bar{W}_{h}\right)\right)>+<\left(\eta_{\alpha} \circ p r_{2} \circ \Phi_{\mathbb{E}}\right)_{*}\left(\bar{V}_{h}\right),\left(\eta_{\alpha} \circ p r_{2} \circ \Phi_{\mathbb{E}}\right)_{*}\left(\bar{W}_{h}\right)> \\
& \left.\left.\left.=<\pi_{*}\left(\bar{V}_{h}\right)\right), \pi_{*}\left(\bar{W}_{h}\right)\right)>_{x}^{\alpha}+<\left(p r_{2} \circ \Phi_{\mathbb{E}}\right)_{*}\left(\bar{V}_{h}\right), p r_{2} \circ \Phi_{\mathbb{E}}\right)_{*}\left(\bar{V}_{h}\right)>_{v}^{\alpha}
\end{aligned}
$$

where $\Phi_{\mathbb{E}}(h)=(x, v)$. At this point we need to note that for all $x \in M$, there exists a diffeomorphism $\gamma_{x}: \pi^{-1}\{x\} \rightarrow F$ with $\gamma_{x}=p r_{2} \circ \Phi_{\mathbb{E}}$, therefore we can rewrite the last equation as follows:

$$
\left.\left.<\bar{V}_{h}, \bar{W}_{h}>_{h}^{\alpha}=<\pi_{*}\left(\bar{V}_{h}\right)\right), \pi_{*}\left(\bar{W}_{h}\right)\right)>_{x}^{\alpha}+<\left(\gamma_{x}\right)_{*}\left(\bar{V}_{h}\right),\left(\gamma_{x}\right)_{*}\left(\bar{V}_{h}\right)>_{v}^{\alpha}
$$

where $\Phi_{\mathbb{E}}(h)=(x, v)$.

Now we define the induced metric $g_{\mathbb{E}}$ on $\mathbb{E}$ by using Equation 6 . For all $h \in \mathbb{E}$,

$$
\begin{aligned}
& g_{\mathbb{E}}\left(\bar{V}_{h}, \bar{W}_{h}\right) \\
& =\sum_{\alpha \in I}\left(\psi_{\alpha} \circ \pi\right)(h)<\bar{V}_{h}, \bar{W}_{h}>_{h}^{\alpha} \\
& \left.\left.=\sum_{\alpha \in I} \psi_{\alpha}(x)\left(<\pi_{*}\left(\bar{V}_{h}\right)\right), \pi_{*}\left(\bar{W}_{h}\right)\right)>_{x}^{\alpha}+<\left(\gamma_{x}\right)_{*}\left(\bar{V}_{h}\right),\left(\gamma_{x}\right)_{*}\left(\bar{V}_{h}\right)>_{v}^{\alpha}\right) \\
& =\sum_{\alpha \in I} \psi_{\alpha}(x)<\pi_{*}\left(\bar{V}_{h}\right), \pi_{*}\left(\bar{W}_{h}\right)>_{x}^{\alpha}+\sum_{\alpha \in I} \psi_{\alpha}(x)<\left(\gamma_{x}\right)_{*}\left(\bar{V}_{h}\right),\left(\gamma_{x}\right)_{*}\left(\bar{W}_{h}\right)>_{v}^{\alpha} \\
& =g_{M}\left(\pi_{*}\left(\bar{V}_{h}\right), \pi_{*}\left(\bar{W}_{h}\right)\right)+\sum_{\alpha \in I} \bar{\psi}_{\alpha}(v)<\left(\gamma_{x}\right)_{*}\left(\bar{V}_{h}\right),\left(\gamma_{x}\right)_{*}\left(\bar{V}_{h}\right)>_{v}^{\alpha} \\
& =g_{M}\left(\pi_{*}\left(\bar{V}_{h}\right), \pi_{*}\left(\bar{W}_{h}\right)\right)+g_{F}\left(\left(\gamma_{x}\right)_{*}\left(\bar{V}_{h}\right),\left(\gamma_{x}\right)_{*}\left(\bar{W}_{h}\right)\right),
\end{aligned}
$$

which ends the proof.

Definition 2. We call $g_{\mathbb{E}}$ in Equation 4 as induced Riemannian metric on $\mathbb{E}$.

Acknowledgements. Hulya Kadioglu would like to thank the Idaho State University Department of Mathematics for being such nice hosts during her visit between August 2015- September 2016. She also acknowledges library support from ISU.

\section{References}

[1] Dold, A., Partitions of Unity in the Theory of Fibrations, Annals of Math, 78 (2), pp: 223-255, 1963.

[2] Lovett, S., Differential Geometry of Manifolds, AK Peters, Ltd., Natick, Massachusetts, 2010.

[3] Lee, J. M., Introduction to Smooth Manifolds, Springer Science+Business, Newyork, 2003. 
[4] Melenk, J. M. and Babuska, I, The Partition of Unity Finite Element Method: Basic Theory and Applications, Computer Methods in Applied Mechanics and Engineering, 139 (No:1-4) 289-314, 1996.

[5] Morimoto, A., Prolongations of G-Structures To Tangent Bundles, Nagoya Math. J., 12, pp: 67-108, 1968.

[6] Pemantle, R. and Wilson, M.C, Asymptotic expansions of oscillatory integrals with complex phase, Contemporary Mathematics: 520, pp: 220- 240, 2010.

[7] Richter, Christian, A chain of controllable partitions of unity on the cube and the approximation of Holder continuous functions, Illinois J. Math. : 43 (1), pp: 159-191, 1999.

[8] Saunders D.J., The Geometry of Jet Bundles, Cambridge University Press, Cambridge-New York, 1989.

[9] Walschap, Gerard, Metric Structures in Differential Geometry, Springer-Verlag, New York, 2004. 